\title{
Rat reasoning: A reliability and validity study
}

\author{
BRITT ANDERSON \\ University of Alabama in Birmingham, Birmingham, Alabama
}

\begin{abstract}
Reasoning is a process that permits solving novel problems by allowing items of old information to be combined into new information. Reasoning, where noncontiguously learned behaviors are combined, is different from learning, where contiguously learned behaviors are combined. To assess whether reasoning could be reliably and validly measured in the adult rat, rats were tested on a variation of the radial eight-arm maze designed to measure reasoning. Performance in this task was consistent across days $(\alpha=.82)$ and led to a consistent ranking of the relative abilities of normal unlesioned adult rats. Performance on the reasoning task did not correlate with reliable measures of emotionality or exploration derived from an open-field test. Reasoning performance did correlate with a reliable measure of preference to novelty (Pearson $r=0.58$, $p=.02$ ); an analogous measure in human infants is known to correlate with subsequent IQs. Thus, the described rat-reasoning measure reliably discriminates performance of normal adult rats, a performance that is not largely determined by emotionality but which is correlated to preference for novelty.
\end{abstract}

So often it is implied that reasoning ability resides only in human beings. Since human beings make this claim, we may question its objectivity. I do not believe psychological terms should be restricted to a certain species. Rather a psychological term should designate a process and different processes should be designated by different terms.

N. R. F. Maier (1937)

Reasoning is the process whereby old information is converted to new information and used for solving novel problems (Sternberg, 1982). Reasoning is critical to impressions about human intelligence (Kail \& Pellegrino, 1985), important to our measurement of human intelligence (Sternberg, 1982), and an important deficiency in people with mental retardation (Campione \& Brown, 1979). Thus it would be interesting and practical to learn more about the brain mechanisms that contribute to the quantitative variation of this capacity.

Since research on humans is limited technically and ethically, it would be beneficial to have an animal model of reasoning. The definition of human reasoning does not require that the information to be manipulated have a linguistic component, so there is no a priori reason why animals cannot reason. Indeed, qualitative work on animal behavior suggests that animals do reason (Epstein, Kirshnit, Lanza, \& Rubin, 1984; Kohler, 1925/1973; Maier, 1929; Yerkes \& Learned, 1925).

The purpose of this project was to see if an animal task could be designed that met the definition of a reasoning test and for which reliability and validity could be dem-

This work was supported by NIA Grant P30-AG10163-01 and by the Department of Neurology, University of Alabama at Birmingham. Lindy E. Harrell provided helpful advice and criticism. Appreciation to Miriam Cobern for typing the manuscript. Correspondence should be addressed to B. Anderson, Department of Neurology, University of Alabama at Birmingham, UAB Station, Birmingham, AL 35294-0007. onstrated, thus making it useful for further correlative studies on the brain mechanisms that contribute to the quantitative variation in reasoning ability. By using rats and a procedural variation of the radial eight-arm maze, I hoped to establish a procedure that could be widely applied. Reliability concerns the extent to which a test yields the same result on repeated trials (Carmines \& Zeller, 1979). To document reliability, the task should allow sufficient variation to permit repeated testing on unique but similar problems and the rats tested on this series of problems should show a consistent ranking of their relative abilities. Validity is the extent to which a test measures what it says it is measuring. Validity can be considered to be of three types: criterion, content, or construct validity. Criterion validity reflects how well test performance predicts or correlates with an independent assessment of the relevant criterion. In this case, that criterion would be reasoning. Since there is no established measurement for reasoning in the rat, criterion validity cannot be determined. Content validity reflects the extent to which the test reflects the content of the criterion to be measured; for example, a math test should be composed of more than just addition problems to be content valid. Content validity has no standard form of measurement, and establishing content validity usually rests on an appeal to reason rather than on empirical measurement. Although I will seek to persuade that the rat reasoning test described is composed of reasoning content, there will be no way to make this case stronger than by arguing from analogy. Construct validity generally reflects the degree to which one measure theoretically believed to relate to the criterion correlates with another measure believed to relate to the relevant criterion. As a practical matter, establishing construct validity often requires examining the correlation between two independent test instruments believed to be measuring the same thing. To apply this to rat 
reasoning would require that the test to be described below be compared to other tests believed to measure reasoning in the rat. There are no other tests that assess rat reasoning beyond Maier's Three Table Problem, and that test is sufficiently similar to the test under discussion to make it problematic whether the two test instruments could be argued to be independent. Thus, we are quite limited to the degree to which we can examine validity for a measurement of rat reasoning. This initial work focuses on content validity. If this attempt is deemed generally acceptable, then it will provide a measure against which subsequent test instruments can be compared in a further effort to expand the validity of rat reasoning measurement to include construct validity. Therefore, to evaluate validity, the reasoning test was constructed such that the rat was required to unite previous experiences (old information) to yield a solution to a novel problem (new information). By showing that rats with only one or the other prior experience did not solve the test as quickly or as accurately as rats given both prior relevant experiences, it will be argued that the rat reasoning test is composed of reasoning content, a content different from that measured by learning paradigms. Furthermore, performance was compared with an emotionality measure as derived from the open-field testing and a measure of exploratory tendency, as assessed both by squares crossed in the open field and a preference-for-novelty assessment derived from the open-field test. Since emotionality should not predominantly determine reasoning, but could importantly influence motoric behavior in a maze via nonreasoning mechanisms, it was hypothesized that the emotionality measure would not correlate if the reasoning task were assessing "reasoning." Since preference for novelty in human infants predicts IQ (Fagan \& Montie, 1988), it was predicted that this measure could correlate to rat reasoning as measured by the proposed rat reasoning test.

\section{MATERIALS AND METHODS}

\section{Subjects}

Thirty-two male Long-Evans rats (initial weight $180-200 \mathrm{~g}$ ) were used. They were housed communally in a temperature- and lightcontrolled (12:12-h light:dark cycle) colony room. When food deprivation was begun, the rats were transferred to individual hanging cages. Food deprivation produced a $20 \%$ decrease in weight. A 5\%-per-week weight increase was allowed. During the 1st week, the animals were handled daily. During the next 2 weeks, the animals were tested in the open-field chamber. Next, following a week when food deprivation was begun, the animals spent 3 weeks being given the reasoning test. Testing was done between 8:00 a.m. and 12:00 p.m.

\section{Behavioral Procedures}

Open field. The open-field chamber was $0.9 \mathrm{~m}$ on a side, $0.5 \mathrm{~m}$ high, and constructed of unfinished plywood. The floor was a plain tile floor divided into nine equal-sized squares $0.3 \mathrm{~m}$ on a side. Testing was conducted during the morning hours under fluorescent lighting. The rats were brought in their home cages to the test room. Each animal was placed in the corner nearest the examiner. For the next $3 \mathrm{~min}$, the number of squares entered by the rat with both forepaws was counted. Also, the number of fecal boli deposited by the rat was counted. The floor was cleansed with a disinfectant/ deodorizer before the first animal and after each animal. The number of fecal boli across the first 4 days for cach animal was computed as a measure of emotionality. The total number of squares entered across the first 4 days was computed as a measure of exploration. The selection of these two measures as representing emotionality and exploration was based on previous reports (Dennenberg, 1969; Kitaoka \& Fujita, 1991; Pradhan \& Arunasmitha, 1991).

On Day 7 in the open field, the amount of time spent in the center square was recorded. On Days 8 and 9, a red magic marker was taped to the center square. On Days 10 and 11, a soda pop can was affixed to the center square. On all these days, the time in the center square was recorded. Figure 1 shows the mean time that the rats spent in the center square across the 5 days and confirms the preference for novelty exhibited on Days 8 and 10. For each animal, the time spent in the center square on Days 8 and 10 was summed to provide the preference-for-novelty measure.

Radial eight-arm maze. The test room was the same room used for open-field testing and contained numerous landmarks, including two desks, a bookshelf, a window, a door, and several chairs. The eight-arm maze was constructed of wood and painted black. The central octagon was $30 \mathrm{~cm}$ across. Each arm was $75 \mathrm{~cm}$ long, $11.75 \mathrm{~cm}$ wide, and lined by $12.7 \mathrm{~cm}$ sides. Both ends were open. The apparatus was $48 \mathrm{~cm}$ off the floor.

The rats were transferred to individual hanging cages, and a food deprivation schedule was begun. The animals were started on this task at $80 \%-85 \%$ of their initial weight. Six days after completion of the open-field measures, training was begun on the reasoning task. The 32 rats were divided into three groups. Group $1(n=$ 20) was the test group. Group $2(n=6)$ and Group $3(n=6)$ were Control Groups 1 and 2 , respectively.

Experience 1: Exploration of the maze. The animals were brought to the test room in groups of 5 or 6 in a plastic cage. Groups of 5 or 6 animals were placed in the center platform and allowed to explore the unbaited maze for $10 \mathrm{~min}$.

Experience 2: Demonstration of the goal arm. Next, the arm selected as the goal arm was baited at its distal end with half a Froot Loop in a recessed cup. A rat was individually placed near the food

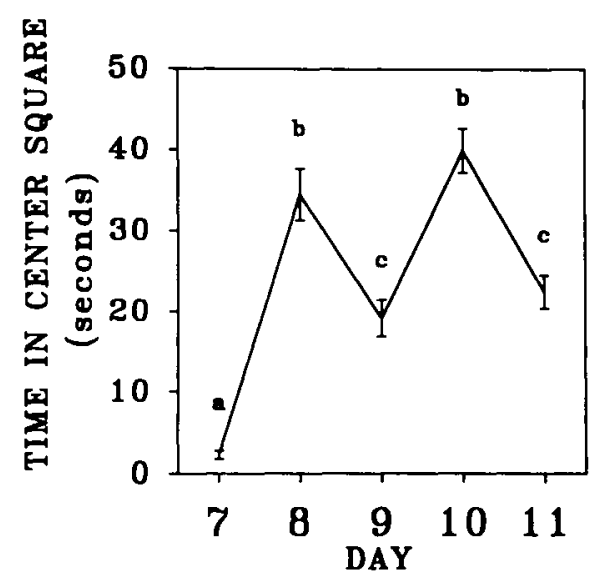

Figure 1. The time spent in the center square of an open field was recorded for Days 7-11. On Days 8 and 9, a magic marker was placed in the center square. On Days 10 and 11, a soda pop can was placed in the center square. An ANOVA showed significant differences between the days $[F(4,155)=39.47, p<.001]$. The mean time for each day is shown \pm 1 SEM. The letters $a, b$, and $c$ denote days differing at the $p<.05$ level by Scheffe's post hoc test. It was demonstrated that exposure to novel objects in the center square (Days 8 and 10) resulted in a significant preference for novelty. 
and allowed to eat. Access to the rest of the maze was prevented by a block at the intersection of the arm and central platform.

Reasoning test: Training. After eating for $30 \mathrm{sec}$, the animal was picked up and carried to the start arm and placed facing out at the distal end. The time to progress halfway down the rebaited goal arm was recorded, as were errors. An error was entering any arm, other than the goal arm, with all four paws. Immediately following completion, the goal arm was rebaited and the run repeated. Group 2 did not receive the $10 \mathrm{~min}$ of unbaited exposure at any time during training or testing, and during the training phase the rats in Group 2 were not started at the start arm but were simply fed on the goal arm three times with handling between each feeding. Thus, they had equal feeding and handling and an equal opportunity to view the room from each arm of the maze, but this group did not have an equal exploratory experience or opportunity to develop a cognitive map. Group 3 had all the experiences except that these rats were not fed on the goal arm prior to being placed on the start arm. Thus, this group had an exploratory phase but was not shown which goal arm was baited.

The purpose of the training phase was to familiarize the animals with the components of the procedure prior to analyzing their performance. After 3 days of the training portion, almost all the animals would work the maze. After 5 days, 6 rats were eliminated from the procedure, 4 in Group 1 and 1 each in Groups 2 and 3. These proportions were equivalent. The eliminated animals either would not eat the food or would not move in the maze, thus making the interpretation of their performance problematic. The arms were labeled clockwise. The pattern of start and goal arms for the training sessions was $4: 1,6: 3,8: 6,1: 2,3: 5,4: 7,2: 4$, and 7:8.

Reasoning test: Testing. The test procedure was the same as that described above for training. All animals were kept in the same groups, and the ordering of the groups rotated each day. Within each group, the ordering of the animals was rotated on a daily basis also. The test portion comprised 8 days of one two-trial session per day. The order of start and goal arms for the test sessions was $1: 3,3: 4,6: 7,2: 5,5: 2,7: 8,4: 1$, and 8:6. The results were recorded in two ways: (1) as the number of test runs out of eight on which the rat erred and (2) as the sum of the reciprocals of the times for each of the eight test runs. The times were used as reciprocals to minimize the effect of rare large values. This is an appropriate method of transformation for data where most values are small and there are occasional large outlying values (Armitage \& Berry, 1987).

\section{Data Analysis}

Correlation analyses were performed using the Pearson productmoment correlation. Reliability was computed using Cronbach's $\alpha$. This method uses the average interitem correlation. This is not a test of significance. Satisfactory reliability estimates, for human behavioral tests, are frequently considered to be greater than .8 . This value is arbitrary, just as the commonly used $p=.05$ is arbitrary (Carmines \& Zeller, 1979). As a significance test of reliability, Kendall's coefficient of concordance $(W)$ was calculated. The distribution of $W$ approximates a chi-square distribution and permits a probability determination (Siegel \& Castellan, 1988). Additionally, analyses of variance with post hoc testing by the method of Scheffé were applied to aspects of the data.

\section{RESULTS}

The reliability for a single measure of emotionality derived from the fecal bolus counts on Days 1-4 in the open field was $\alpha=.65$. Kendall's $W$ (corrected for ties) was 0.49 (df $=31, p<.001$ ), demonstrating significant concordance for the ranking of the rats by number of fecal boli for each of the 4 days. The same measures for the index of exploration were $\alpha=.74$ and $W=0.54(d f=$
$31, p<.001)$. There was no significant correlation between the squares crossed and the fecal boli measure $(r=$ -.002 ). The emotionality measure correlated with the preference-for-novelty measure, which might be expected since the rats had to go into the center square to attend to the object and more timid rats are less likely to enter the center square of the open field $(r=-.609, d f=15$, $p=.01$ ).

As shown in Figure 1, the mean time spent in the center square varied across days $[F(4,155)=39.47, p<.001]$. Post hoc testing showed that the 4 days when an object was present in the center square all showed an increase in time in the center square over baseline. The 2 days when a novel object was first introduced did not differ from each other but were significantly greater than the second exposures to each object. By demonstrating an increased response over baseline and a decrement on repetition, a preference for novelty was demonstrated. The correlation for the two exposures to a novel object was $r=0.59(d f=31, p<.001)$. The reliability was $\alpha=$ .74. Although the group as a whole demonstrated habituation, the time spent in the center square on the second exposure to each object was significantly correlated with the time spent in the center square on the first exposure to the object. Furthermore, given the different starting times for individual animals, the use of percent changes to represent a measure of habituation was problematic. That is, should the change from $12 \mathrm{sec}$ to $8 \mathrm{sec}$ be regarded as equivalent to the change from $60 \mathrm{sec}$ to $40 \mathrm{sec}$ ? For this reason, it was not possible to derive a measure of habituation from this data to be applied in later analyses, and only a preference-for-novelty measure was computed and used.

The reasoning test showed significant differences between the test group and each of the two control groups (Figures 2A and 2B) for both error $(p>F=.0001$, $d f=2,123, F$ ratio $=38.72$, Scheffé $p<.05)$ and time measures $(p>F=.0002, d f=2,123, F$ ratio $=$ 12.85 , Scheffe $p<.05$ ). The reliability of the reasoningtime measure for the test group was $\alpha=.82$, and there was a significant concordance $(W=0.45, d f=15$, $p<.001$ ).

There was no correlation in the test group between the time measure and the measure of emotionality or exploration derived from the open field or for the error measures and either exploration or emotionality in the open field. The correlation between the time measure and the error measure was $r=-0.485(d f=15, p=.06)$. The correlation between the preference-for-novelty measure and the reasoning-time measure was $r=0.58(d f=15, p=$ .02 ), and between the preference-for-novelty measure and the error measure it was $r=0.49(d f=15, p=.06)$.

Additionally, there was no correlation between reasoning performance and the degree of weight loss.

\section{DISCUSSION}

The purpose of this investigation was to examine the validity and reliability of a proposed rat reasoning test. 
A.
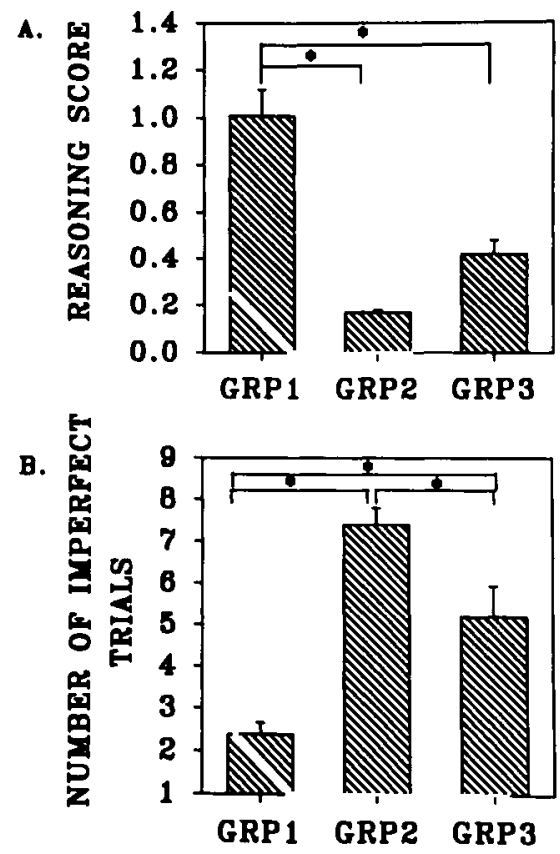

Figure 2. Ressoning-test performance of the test group (GRP 1), the control group with no exploratory experience (GRP 2), and the control group with no preexposure to the food (GRP 3) are compared. (A) Comparison for the total time to acquire the reward over the 8 days summed as reciprocals. (B) Comparison for the number of trials on which the animals committed at least one error. All results are plotted as the mean +1 SEM. Asterisks deatgrate groups differing at the $p<.05$ level by Schefres poet hoc test. The test group was superior to both control groups on both mensures.

Reasoning is different from learning (Maier, 1937). Learning reflects the improved performance that comes from contiguous exposure to the necessary experiences. Reasoning reflects the improved performance that comes from noncontiguous exposure to, and integration of, the necessary experiences. Therefore, if a test is to measure reasoning, it must require the rat to unite previous experiences to solve the problem, and rats with only one or the other preliminary experience should not perform as well as animals that have had both experiences. The radial eight-arm maze paradigm used here was designed with that precept in mind. It was hypothesized that to find the food quickly and accurately, the rats that had both an exploratory experience and been shown the location of the day's goal arm would perform better than the rats that had only had an exploratory experience or had only seen the location of that day's goal arm. To separate the reasoning paradigm from a learning measure, it was constructed so that the animals had not been reinforced for running from a particular start arm to a goal arm prior to the reasoning test. This can be seen from an example. Consider the situation where the start arm was Arm 2 and the goal arm was Arm 5. Since the maze is unbaited during the exploratory period, the rat may well go from Arm 2 to Arm 5, but it has not been reinforced for this behavior.
After the animal has been fed on Arm 5, it knows the goal location. When being started at Arm 2, it must unite its knowledge of where to go with how to get there. If the rat responds with a quick errorless run, it must have reasoned where to go, since it cannot have learned where to go. By the daily variation in start and goal locations, the animal cannot learn a particular route to food, but for consistent performance it must reason out each day's response.

As empirical confirmation of this view, it was demonstrated that the rats with both the exploratory experience and the pretest exposure to food performed better than the rats deprived of one or the other experience (Figure 2). This confirmed that optimal performance depended on exposure of the rat to both experiences. It may seem obvious that the control group without the exploratory experience failed to solve the problem quickly, perhaps because lacking exploration, these rats have not been able to form a cognitive map or, since some question the cognitive mapping theory, they were limited in the ability to form associations between extramaze cues and goal-arm identities (Brown, 1992). It may also seem obvious to some that the group that was allowed to explore, and through the training sessions was able to learn to associate the maze with the presence of reward, but was not shown the location of that day's goal arm would also be slower and less accurate than the test rats. Although the fact that both control groups would do worse seemed likely before beginning, this presumption did not eliminate the need for an empirical demonstration. Since the animals could have solved the maze using cues, such as olfactory ones, that may not have been apparent to the experimenter, it was deemed necessary to prove that both experiences thought to be necessary for optimal solution were indeed required. Furthermore, whether the control rats failed to solve the maze as quickly as the test rats because the control groups did not have an ample chance to develop a cognitive map or develop associations between particular goal arms and extramaze cues is essentially irrelevant to the question that was being examined here, which was, regardless of the content provided by the two experiences, are both necessary to solving the problem in an optimal fashion? If so, and it was so demonstrated, then the test rats must be integrating those two experiences to solve the novel problem optimally. If this seems obvious, then it should be similarly obvious that rats reason.

To evaluate whether this reasoning test could be used as an independent variable in future correlative studies on the biological basis of reasoning, it was necessary to document the reasoning test's reliability. This was done by showing that for normal adult male rats, the test reliability was $>.8$ and also that the 8 test days were generally concordant in their ranking of the performance of individual rats. It should be emphasized, though, that reliability of a test does not equate with validity.

For validity determination, factors were evaluated that either would or would not be expected to correlate if the test measured reasoning. Emotionality is a factor that 
might be expected to influence performance in a maze task but would be considered distinct from reasoning. For the emotionality measure, there was no significant correlation to the reasoning measures. This suggests that for male Long-Evans rats willing to submit to the reasoning task, emotionality is not the primary determinant of individual variation in performance.

As an additional assessment of validity, we compared the reasoning performance to measures of exploration, the number of squares crossed in the open field and a measure of preference for novelty. It is a well-recognized fact that laboratory rats show a tendency to approach novel aspects of a familiar environment (Barnett, 1975). The data presented here show that there was a consistent individual variation for this tendency in the rats and that their preference for novelty correlated with the reasoning measures. The importance of this point is that a similar measure has been shown in human infants to correlate with subsequently measured IQs. Thus, the candidate rat reasoning test correlated to an external measure assumed to relate to human reasoning. This is not to argue that preference for novelty causes better reasoning in rats, merely that the rat reasoning test may assess brain mechanisms in a similar way to human reasoning tests since both correlate with a similar external measure.

The measures used to assess performance on the reasoning test included both a time measure and an accuracy measure. The decision to use both types of measures was made because it seemed intuitively plausible that a quick accurate response might indicate something different from slow accurate responses. The results here show that there is some repetition between the two measures since nearly $25 \%$ of the variability in each can be explained by common factors. This is somewhat expected, since rats that make errors will have to spend additional time running to extra potential goal arms. However, not all the difference in reasoning-time scores can be explained by differences in error scores. Since this study was designed primarily with reliability and, as much as possible, validity considerations paramount, it was elected not to bias the reporting method toward time or error measures but to include results of both. Which measure is preferable, or if both are complementary, cannot be determined from this study, but will require additional investigation.

The methods by which rats reason have not been clearly established by this investigation. Rats could reason by the enhanced ability to develop associations provided by exposures to the relevant experiences, or it may be that rats are able to use a cognitive map developed during the exploratory phase to plot a quick accurate route to that particular day's goal. The description of a reliable method for measuring this ability should permit further dissection of the psychological mechanisms utilized by the reasoning rat.

The basic arguments of this work are that humans reason and that their reasoning contributes to intelligent behavior; animals also reason, and the test described herein should theoretically assess this process. Furthermore, the described test is shown here to have empirical support as a reasoning test, is shown to be a reliable test, and has been partially validated as a measure of reasoning since performance on the reasoning test is not predominantly influenced by basic drives, but does correlate to a measure assumed to relate to human reasoning. Therefore, the described rat reasoning test may be useful for the purpose of assessing the brain factors that determine the normal variation observed for reasoning ability.

\section{REFERENCES}

ARmitAge, P., \& BerRy, G. (1987). Statistical methods in medical research (2nd ed.). Oxford: Blackwell.

BARNeTT, S. A. (1975). The rat: A study in behavior. Chicago: University of Chicago Press.

Brown, M. F. (1992). Does a cognitive map guide choices in the radialarm maze? Joumal of Experimental Psychology: Animal Behavior Processes, 18, 56-66.

CAmpione, J. C., \& Brown, A. L. (1979). Toward a theory of intelligence: Contributions from research with retarded children. In R. J. Sternberg \& D. K. Detterman (Eds.), Human intelligence: Perspectives on its theory and measurement (pp. 139-164). Norwood, NJ: Ablex.

Carmines, E. G., \& Zeller, R. A. (1979). Reliability and validity assessment. Beverly Hills: SAGE.

DenNenberG, V. H. (1969). Open field behavior in the rat: What does it mean? In E. Tobach (Ed.), Experimental approaches to the study of behavior (Annals of the New York Academy of Sciences, Vol. 159, pp. 852-859). New York: New York Academy of Sciences.

Epstein, R., Kirshnit, C. E., Lanza, R. P., Rubin, L. C. (1984). "Insight" in the pigeon: Antecedents and determinants of an intelligent performance. Nature, 308, 61-62.

FAGAN, J. F., III, Montie, J. E. (1988). Behavioral assessment of cognitive well-being in the infant. In J. F. Kavanagh (Ed.), Understanding mental retardation (pp. 207-221). Baltimore: Brookes.

Kail, R., Pellegrino, J. W. (1985). Human intelligence: Perspectives and prospects. New York: W. H. Freeman.

KitAoKA, A., \& FujrtA, O. (1991). Behavioral comparisons of the Tsukuba emotional strains of rats (Rattus norvegicus) in three types of novel situations. Behavior Genetics, 21, 317-325.

KoHLER, W. (1973). The mentality of apes. London: Routledge \& Kegan Paul. (Original work published 1925)

MaIer, N. R. F. (1929). Reasoning in white rats. Comparative Psychological Monographs, 6, 1-93.

MAIER, N. R. F. (1937). Reasoning in rats and human beings. Psychological Review, 44, 365-377.

Pradhan, N., \& Arunasmitha, S. (1991). Correlations of motility, defecatory behavior and striatal dopaminergic activity in rats. Physiology \& Behavior, 50, 135-138.

Siegel, S., \& CASTEllaN, N. J., JR. (1988). Nonparametric statistics for the behavioral sciences (2nd ed.). New York: McGraw-Hill.

STERNBERG, R. J. (1982). Reason, problem solving, and intelligence. In R. J. Sternberg (Ed.), Handbook of human intelligence (pp. 225307). Cambridge: Cambridge University Press.

Vietze, P. M., \& Contes, D. L. (1986). Information-processing approaches to early identification of mental retardation. In $\mathbf{A}$. $M$. Wisniewski \& D. A. Snider (Eds.), Mental retardation: Research, education, and technology transfer (Annals of the New York Academy of Sciences, Vol. 477, pp. 266-276). New York: New York Academy of Sciences.

YeRKES, R. M., LEARNED, B. W. (1925). Chimpanzee intelligence and its vocal expressions. Baltimore: Williams \& Wilkins.

(Manuscript received January 6, 1992; revision accepted for publication June 15, 1992.) 